\title{
Efficacy of a Single Sequence of Intermittent Bright Light Pulses for Delaying Circadian Phase in Humans
}

\author{
Claude Gronfier ${ }^{1}$, Kenneth P. Wright Jr. ${ }^{1,2}$, Richard E. Kronauer ${ }^{3}$, \\ Megan E. Jewett ${ }^{1}$, and Charles A. Czeisler ${ }^{1}$ \\ ${ }^{1}$ Division of Sleep Medicine, Brigham and Women's Hospital, \\ Harvard Medical School, Boston, MA 02115 \\ ${ }^{2}$ Department of Integrative Physiology, Center for Neuroscience, \\ University of Colorado, Boulder, CO 80309 \\ ${ }^{3}$ Harvard University, Pierce Hall, Cambridge, MA 02138
}

Running head: Phase delaying efficacy of intermittent bright light

\author{
Contact information: \\ Claude Gronfier, Ph.D. \\ INSERM - U371 \\ 18 Avenue du Doyen Lépine \\ 69675 Bron Cedex - France \\ Phone: (+33) 4.72.91.34.89 \\ Fax: $\quad$ (+33) 4.72.91.34.61
}




\section{ABSTRACT}

It has been shown in animal studies that exposure to brief pulses of bright light can phase shift the circadian pacemaker, and that the resetting action of light is most efficient during the first minutes of light exposure. In humans, multiple consecutive days of exposure to brief bright light pulses have been shown to phase shift the circadian pacemaker. The aim of the present study was to determine if a single sequence of brief bright light pulses administered during the early biological night would phase delay the human circadian pacemaker. Twenty-one healthy young subjects underwent a $6.5 \mathrm{~h}$ light exposure session, in one of three randomly assigned conditions: 1) continuous bright light of $\sim 9,500$ lux; 2) intermittent bright light (six 15-min bright light pulses of $\sim 9,500$ lux separated by 60 min of very dim light of $<1$ lux); 3) continuous very dim light of $<1$ lux. Twenty subjects were included in the analysis. Core body temperature (CBT) and melatonin were used as phase markers of the circadian pacemaker. Phase delays of CBT and melatonin rhythms in response to intermittent bright light pulses were comparable to those measured after continuous bright light exposure, even though the total exposure to the intermittent bright light represented only $23 \%$ of the $6.5 \mathrm{~h}$ continuous exposure. These results demonstrate that a single sequence of intermittent bright light pulses can phase delay the human circadian pacemaker and shows that intermittent pulses have a greater resetting efficacy on a per minute basis than does continuous exposure.

Keywords: intermittent light, melatonin, circadian pacemaker, photoreception, phase shift 


\section{INTRODUCTION}

The daily alternation of light and darkness is the primary synchronizer of endogenous circadian oscillators to the 24-hour day. The role of light as a phase resetting agent of the human circadian pacemaker has been demonstrated in a variety of experiments using single or multiple sequences of continuous bright light exposure (for review see ref. (17)). The phase-shifting effect of bright light on the human pacemaker has been shown to be phase-dependent (33). Exposure to several hours of bright light for up to 3 consecutive days have been found to reset the endogenous circadian rhythms of core body temperature, cortisol, melatonin, alertness, performance and TSH by up to 12 hours $(1,9,12,14-16,18,19,21,24,25,30,48-50,55)$. Moreover, it has been shown that even room light can significantly reset the phase of the human circadian clock $(5,6)$. In fact, Zeitzer et al. (56) found that half of the maximum phase delay resetting response achieved by a single exposure to bright light ( 9,000 lux $)$ during the early biological night can be obtained by $\sim 1 \%$ of this light intensity, i.e., ordinary room light of $\sim 100$ lux.

It has been reported in studies of nocturnal animals that the mammalian circadian system is responsive to brief exposures of light. Nelson and Takahashi (43) demonstrated that pulses of bright light as short as 3 seconds produced measurable phase shifts in the golden hamster. They also showed that extending the duration of the stimulus beyond 5-min produced little additional phase shift. Furthermore, they found that the shift obtained by two 5-min light pulses separated in time by up to one hour produced a phase resetting response similar to that of a single 5-min pulse (42). Others have reported that the circadian system can be reset by a chain of very brief light pulses of 2 milliseconds in mice (51), and 10 microseconds in duration in rat (2). The 
structure of the physiological system responsible for such a dynamic resetting of the circadian pacemaker is not yet known. They have been hypothesized by Kronauer and colleagues $(38,39)$ to involve light sensitive elements that are set to a "used" state after being exposed to light, and slowly recover their initial "ready state" after a period of recycling after light removal.

To date there has been few systematic evaluation of the relative effectiveness of different durations of light exposure in the human circadian system (25), although the results of studies on the resetting response to intermittent bright light exposure suggest that humans are also responsive to brief pulses of light. Indeed, three consecutive days of exposure to six 40-min pulses of bright light $(\sim 5,000$ lux $)$ have been shown to phase delay circadian rhythms in humans (3). In other studies, three to five consecutive days of exposure to intermittent pulses of bright light (3000 to $11,000 \mathrm{lux}$ ) as brief as $5 \mathrm{~min}$ in duration have been shown to induce phase advances of the endogenous circadian pacemaker in humans $(10,11,45)$. These findings suggest that humans, like animals, are sensitive to brief bright light pulses. However, interpretation of the temporal dynamics of the resetting response was complicated by the use of multiple, consecutive days of light exposure in those studies.

In the present study, we therefore quantified the phase-resetting efficacy of a single sequence of intermittent bright light pulses given during the biological night, as compared to subjects exposed to continuous bright light or to very dim light at the same circadian phase. Based on prior results, we anticipated a robust phase delay in the groups of subjects exposed to both continuous (33) and intermittent (45) bright light vs. small drifts in phase in the group of control 
subjects exposed to continuous very dim light due to the near-24-h period of the human circadian pacemaker $(13,33,34,54)$. In addition, based on a mathematical model of the effect of light on the human circadian pacemaker developed by Kronauer and co-workers $(32,38,39)$, we predicted that the resetting effect of the intermittent light exposure would be more efficient than continuous bright light exposure, as measured by the amount of shift induced per minute of bright light exposure.

\section{MATERIAL AND METHODS}

\section{Subjects}

Twenty-one healthy subjects participated in the study; 20 subjects $[24.3 \pm 3.9$ (SD) years old, 15 males, 6 females, BMI $22.8 \pm 2.3$ (SD)] were included in the analysis due to non-compliance to constant routine procedures by one subject. The subjects had no medical, psychiatric, or sleep disorders as determined by history, physical examination, electrocardiogram, blood and urine biochemical screening tests, and psychological screening questionnaires (Minnesota Mutliphasic Personality Inventory and Beck Depression Inventory). A staff psychologist interviewed subjects and those with a history of or a current psychiatric pathology were excluded. Subjects reported that they were not taking any medication and were instructed to abstain from the use of alcohol, nicotine, recreational drugs, and foods or beverages containing caffeine for three weeks prior to the study. All participants were drug free at the time of study as verified by a comprehensive toxic analysis conducted upon admission to the laboratory. All experimental procedures were carried out in accordance with the principles of the Declaration of Helsinki, and the protocol was 
approved by the Human Research Committee at the Brigham and Women's Hospital. Prior to beginning the protocol, all participants gave informed, written consent.

\section{Overall study design}

Subjects were required to maintain a regular 8:16 h sleep: wake schedule at home for at least 3 weeks prior to admission to the laboratory. In order to ensure compliance to this protocol, subjects were required to call into a date/time-stamped answering machine just prior to going to bed and immediately upon awakening, and the times were compared to sleep-wake logs on the day of admission. In addition, wrist activity and light exposure were monitored for 1 week immediately prior to admission to the laboratory (Actiwatch-L, Mini Mitter, Sunriver, OR, USA) and were used to verify the stability of their sleep-wake cycle during that last week and throughout the entire impatient protocol.

Upon admission to the study on experimental day 1, subjects were maintained in an environment free from external time cues, including clocks, radios, television, visitors, and sunlight. Subjects maintained contact with staff members specifically trained to avoid communicating time of day or the nature of the experimental conditions to the subjects. Subjects were adapted to the laboratory with three baseline days (Days 1-3), during which time they continued to sleep and wake at their habitual times (Fig. 1). In order to assess their endogenous circadian phase before the light stimulus and to appropriately center the light stimulus during the following scheduled episodes of wakefulness, subjects underwent a 26.2-h initial constant routine (CR1; procedure described below) from day 4 to day 5 . The duration of the constant routine-and the associated 
shift of the sleep episodes 4 and 5 (see Figure 1) —was designed so that the 6.5-h light exposure session (centered in the middle of the wake period) would coincide with the delay portion of the human phase response curve (33), and was started $9.05 \mathrm{~h}$ before habitual bedtime and ended 2.55 $\mathrm{h}$ before habitual wake time (corresponding to a center at $\sim 3.5 \mathrm{~h}$ before the subjects' core body temperature minimum [CBTmin]).

On day 5, subjects were randomly assigned to one of the three light exposure conditions (described below). Then, they were scheduled to a final constant routine of 64-h duration (Days 6-9) to re-assess their endogenous circadian phase and thereby estimate the phase shift from the initial constant routine. Subjects were then discharged on day 10, after a $\sim 22$-h recovery sleep episode.

During wake episodes, subjects were free to move about the suite as desired, except that they were asked not to lie down, nap, or exercise. Subjects' compliance with the protocol was monitored by means of closed-circuit cameras and frequent interaction with technicians.

The experimental suites were equipped with ceiling-mounted cool-white $(4,100 \mathrm{~K})$ fluorescent lamps [Phillips (Eindhoven, The Netherlands) T8 and T80]. A computer system automatically turned the lighting to the required pre-set intensity at the scheduled times. Maximum light intensities, as measured vertically at a height of $\sim 1.83 \mathrm{~m}$ using an IL1400 photometer (International Light, Newburyport, MA), were 190 lux during the waking hours of the three baseline days ( $\sim 90$ lux measured horizontally at a height of $\sim 1.37 \mathrm{~m}) ;<8$ lux $(\sim 1.5$ lux measured horizontally at a height of $\sim 1.37 \mathrm{~m}$ ) during the constant routines and the day of light exposure; and depending on the experimental condition (described below), <1 lux ( 0.5 lux measured 
horizontally at a height of $\sim 1.37 \mathrm{~m}$ ) or $\sim 9,500$ lux (in the direction of gaze at a wall-mounted target) during the light exposure session. Sleep episodes were conducted in darkness. The experimental suites were equipped with hand-held terminals for on-line event recording, a porthole for 24-h blood sample collection with minimal sleep disturbance, a video camera and a voice-activated audio system.

\section{Constant Routine Procedure}

The constant routine (CR) procedure was used to assess the phase of two markers of the endogenous circadian pacemaker: the core body temperature rhythm and the melatonin rhythm. The methodology we used involved a refinement of a technique first proposed by Mills et al. (41), according to which the subjects are studied under constant environmental and behavioral conditions in order to eliminate or distribute across the circadian cycle the physiologic responses evoked by environmental or behavioral stimuli, such as sleeping, eating, and changes in posture (22). The CR consisted of a regimen of enforced wakefulness in a semi-recumbent posture in constant dim illumination of $\sim 1.5$ lux. Subjects were required to maintain a very low level of physical activity and were not permitted to change their posture throughout the constant routine. This posture was also maintained for urine samples and bowel movements. Nutritional intake was divided into hourly isocaloric snacks to maintain an equal caloric intake across the circadian cycle. Caloric requirements were calculated with use of the Wilmore nomogram (53) to determine the basal metabolic rate, and adjusted upward by a $10 \%$ activity factor. Fluid intake was calculated for each subject to account for the sedentary nature of the CR. A staff member 
monitored the subject to help maintain wakefulness and to ensure compliance with the posture and activity level requirements.

\section{Light exposure conditions}

Subjects were randomly assigned to one of the three light exposure conditions (Fig. 2). The light exposure session of 6.5 hours duration was centered $5.8 \mathrm{~h}$ before habitual wake time (i.e., it started $\sim 1.1 \mathrm{~h}$ before habitual bedtime and ended $\sim 2.6 \mathrm{~h}$ before habitual waketime) and therefore 3.5 hours before $\mathrm{CBT}_{\min }(23)$. The three conditions were: continuous bright light (BL) of 9,500 lux, defined as 100\% bright light; intermittent bright light (IBL) consisting of six 15minute bright light pulses of $\sim 9,500$ lux separated by 60 minutes of very dim light (<1 lux), defined as $23 \%$ bright light; and continuous very dim light (VDL) of <1 lux, defined as $0 \%$ bright light. In all three conditions, the 30 minutes prior to and the 30 minutes following the light exposure session were conducted in $<1$ lux. Seven subjects were randomized to each condition. Subjects were seated in a chair from 2 hours before until 2 hours after the end of the light exposure session. A technician was present at all times during the light exposure session to ensure compliance with the protocol. During the light exposure sessions, subjects were instructed to fix their gaze on a target mounted on the wall of their suite for 5 of every 10 minutes and then allowed free gaze for 5 of every 10 minutes to ensure consistency of light exposure between subjects. This alternating fixed and free gaze episodes continued during the entire duration of the light exposure sessions. Light intensity measurements were taken in the direction of gaze every five minutes to ensure that subjects were exposed to the appropriate light intensity for the appropriate duration. Clear polycarbonate lenses filtered $99.9 \%$ of the light in 
the UV range from the light source. In addition, all subjects wore clear goggles (model \#S379; luminous transmittance 90\%, UV absorption >99\%, Uvex Safety, Smithfield, RI, USA) during the exposure sessions to bright light for additional protection against UV exposure.

\section{Data Collection}

Temperature data. A real time, on-line data acquisition system utilizing IBM-PC-compatible computers was employed to monitor and collect temperature data. Core body temperature was continuously recorded via a disposable rectal thermistor (Yellow Springs Instrument, Yellow Spring, OH, USA), and room temperature was recorded by means of a permanent air temperature thermistor; both were recorded every minute.

Hormonal data. Blood samples were collected every 40 minutes during constant routines, through an indwelling intravenous catheter that was inserted into a forearm vein on day 2 of the study, and every 5 to 10 minutes during the light exposure session (data will be presented elsewhere). A solution of heparinized saline ( $0.45 \%$ sodium chloride, 10 units of heparin $/ \mathrm{ml})$ was infused at a slow rate $(20 \mathrm{ml} / \mathrm{h}, 200 \mathrm{IU}$ heparin/h) between blood samples. We provided participants with Ferrous Gluconate $(324 \mathrm{mg}$ ) pills to be taken at breakfast and dinner: 1) for a minimum of one week prior to participation in the inpatient research protocol; 2) during the inpatient portion of the protocol; and 3) for three weeks after completion of the protocol. Subjects' hemoglobin levels were tested every 1-2 day(s) to ensure appropriate levels (>11.0 for men, >10.3 for women). Blood samples were collected in ethylenediaminetetraacetate (EDTA)$\mathrm{K}_{2}$ tubes, rapidly centrifuged at $4^{\circ} \mathrm{C}$, and the plasma was stored at or below $-25^{\circ} \mathrm{C}$ until assayed. 
Samples were assayed for melatonin using radioimmunoassay techniques (Diagnostech / Pharmasan, Osceol WI, USA). The assay sensitivity was $2.5 \mathrm{pg} / \mathrm{ml}$. The average intra-assay coefficients of variation (CVs) were $6.4 \%$ below $50 \mathrm{pmol} / \mathrm{L}$, and $4.9 \%$ above.

\section{Estimation of circadian phase}

For temperature data collected during constant routines, a non-linear least-squares dual-harmonic regression model with correlated noise (8) was used. The time of the fitted core body temperature minimum $\left(\mathrm{CBT}_{\min }\right)$ was defined as the marker of the temperature phase. Data collected during the first 5 hours of the constant routines were excluded to minimize potential masking effects from the preceding sleep episode, or from changes in posture from flat to the semi-recumbent CR posture. In order to minimize uncertainty in the phase estimate, subjects for whom amplitude of the temperature rhythm was below $0.14^{\circ} \mathrm{C}$ were not included in the analysis (24) (see Table 1). In order to minimize the effect of inter-individual differences in circadian period on the phases shift assessments only the first circadian oscillation of melatonin and CBT of the final CR was included in the analysis, and phase was estimated on the same time window length as for CR1.

For melatonin data, a three-harmonics least-square regression analysis was applied to data collected during CR1 (Fig. 3, thick line) to estimate the amplitude of each subject's melatonin rhythm (Fig. 3, dashed line). A threshold of $25 \%$ of the peak-to-trough amplitude of the fitted curve (54) was calculated (Fig. 3, dotted line) and applied to data of CR1 and CR2 (first circadian oscillation considered). Three markers of melatonin phase were computed for each CR 
data: 1) the midpoint $\left(\mathrm{MP}_{25 \%}\right)$ between 2) the upward (DLMOn $25 \%$, and 3) the downward (DLMOff $25 \%$ ) crossings of this $25 \%$ threshold.

Phase shifts of temperature and melatonin rhythms were calculated as the difference between the phase in CR1 and the phase in CR2.

\section{Statistical analysis}

Repeated-measure ANOVA (condition x phase marker) with Greenhouse-Geisser correction (only original degrees of freedom are reported), and Tukey tests for post-hoc multiple comparisons were used to compare phase shifts between phase markers, with $\mathrm{H}_{0}$ : $\mu \mathrm{DLMOn} \mathrm{M}_{25}=$ $\mu \mathrm{MP}_{25 \%}=\mu \mathrm{DLMOff}_{25 \%}=\mu \mathrm{CBT}_{\text {min. }}$. Pearson correlations were calculated between phase shifts and initial phases of light exposure, between $\mathrm{CBT}_{\min }$ and melatonin phases shifts, and between phase shifts of the melatonin phase markers (DLMOn $25 \%$ and DLMOff $25 \%$ ).

\section{RESULTS}

The light stimulus was on average centered $3.15 \pm 0.72 \mathrm{~h}$ after $\operatorname{DLMOn}_{25 \%}$ and $3.39 \pm 1.28 \mathrm{~h}$ prior to $\mathrm{CBT}_{\min }$ (i.e., it started on average 5 minutes $(0.09 \pm 0.72 \mathrm{~h})$ before $\mathrm{DLMOn}_{25 \%}$ and ended 5 minutes $(0.09 \pm 1.56 \mathrm{~h})$ after $\mathrm{CBT}_{\min }$ (Table 1$)$. There were no significant differences between the three groups in the initial phases of the temperature and melatonin rhythms at which the light intervention occurred. There were minimal changes in phase observed in the control group of subjects exposed to very dim light (median phase change of $+0.44 \mathrm{~h}$ for $\mathrm{CBT}_{\min }$ and $0.31 \mathrm{~h}$ for $\mathrm{DLMOn}_{25 \%}$; Fig.4). A significantly greater phase delay was observed in subjects exposed either to continuous bright light [median phase delay $=-2.70 \mathrm{~h}$ for $\mathrm{CBT}_{\min }(\mathrm{p}<0.0002$ vs. 
controls), $3.03 \mathrm{~h}$ for $\mathrm{DLMOn}_{25 \%}$ ( $\mathrm{p}<0.0002$ vs. controls), $-2.71 \mathrm{~h}$ for $\mathrm{MP}_{25 \%}(\mathrm{p}<0.0002$ vs. controls), and $-2.38 \mathrm{~h}$ for $\operatorname{DLMOff}_{25 \%}(\mathrm{p}=0.0003 \mathrm{vs}$. controls)] or intermittent bright light [median phase delay $=-1.73 \mathrm{~h}$ for $\mathrm{CBT}_{\min }\left(\mathrm{p}<0.0003\right.$ vs. controls), $-2.34 \mathrm{~h}$ for $\mathrm{DLMOn}_{25 \%}$ ( $\mathrm{p}<0.0002$ vs. controls), $-2.30 \mathrm{~h}$ for MP25\% ( $\mathrm{p}<0.0002$ vs. controls), and $-1.63 \mathrm{~h}$ for $\mathrm{DLMOff}_{25 \%}$ ( $\mathrm{p}=0.001$ vs. controls)]. As expected for the selected phase of light exposure, the continuous and intermittent bright light groups demonstrated phase delays of their endogenous circadian rhythms of core body temperature and melatonin (Fig. 4). Although the phase shifts observed in the IBL group were slightly smaller than those observed in the BL group, no statistical differences were found for any marker (Fig.4).

The resetting responses measured with $\mathrm{CBT}_{\min }$ were more variable than those measured with melatonin (Table 1), however, we found highly significant and positive linear relationships between the markers of the two rhythms $\left(\mathrm{r}_{\mathrm{CBTmin} / \mathrm{DLMOn25} \%}=0.896, \mathrm{r}_{\mathrm{CBTmin} / \mathrm{MP25} \%}=0.871\right.$, $\mathrm{r}_{\mathrm{CBTmin} / \mathrm{DLMOff25} \%}=0.779 ; \mathrm{p} \leq 0.0001$ for all three correlation coefficients).

We found no significant linear correlation between observed melatonin phase shifts and the initial phases of light exposure ( $\left.\phi_{\text {init }}\right)$ within the limited range of initial phases to which subjects were exposed within each condition $\left(\mathrm{r} \phi_{\text {init/DLMOn25 }}=0.182, \mathrm{r} \phi_{\text {init/MP25\% }}=0.071, \mathrm{r} \phi_{\text {init/DLMff25\% }}=\right.$ 0.031; p non significant for all three correlation coefficients)

In appraising the variability of the phase shift data presented here (Table 1) we recognize three independent variability sources whose variances should sum: 1) noise in the individual phase assessments whose difference comprises the phases shift, 2) inter-subject differences in the 
endogenous circadian period that leads to different phase drifts over the two cycles of shift assessments, 3) inter-subject differences in photic response arising either from sensitivity differences or from differences in the timing of the stimulus. Klerman et al. (36) found that the method of single-phase assessment yielding the lowest variance was a normalized level-crossing on the rising front of the melatonin pulse. This variance could be as low as $0.04 \mathrm{~h}^{2}\left(50^{\text {th }}\right.$ percentile) so that for phase shifts the variance might be $0.08 \mathrm{~h}^{2}$. For our results the DLMOn ${ }_{25 \%}$ does indeed give consistently the lowest variance estimates. Czeisler et al. (13) estimated the standard deviation of circadian period for young adults to be $0.13 \mathrm{~h}$ so that a two-cycle phase drift would show a variance of $0.07 \mathrm{~h}^{2}$. We recognize that variance estimates are extremely unreliable for data groups of 6 or 7 members. However, the value of $0.15 \mathrm{~h}^{2}$ for the VDL condition is consistent with the sum of $0.08 \mathrm{~h}^{2}$ and $0.07 \mathrm{~h}^{2}$ for sources (1) and (2) described above. The variance of $0.20 \mathrm{~h}^{2}$ for the BL condition suggests that perhaps as little as $0.05 \mathrm{~h}^{2}$ might be ascribed to source (3). However, by this reasoning the variance of $0.12 \mathrm{~h}^{2}$ for the IBL condition is inexplicably small and may represent a remarkably homogeneous subject group with little difference in endogenous circadian period.

\section{DISCUSSION}

The current results demonstrate that a single sequence of intermittent bright light exposure is effective in inducing phase delays of the human circadian timing system. The phase delays observed after intermittent bright light exposure were comparable to those measured after continuous bright light exposure (Fig.4), even though the bright light represented only $23 \%$ of the $6.5 \mathrm{~h}$ light exposure session. Given that the median phase delay measured with DLMOn $_{25 \%}$ 
was $-3.03 \mathrm{~h}$ under continuous bright light condition ( 6.5 hours of bright light), and $-2.34 \mathrm{~h}$ in the intermittent bright light ( $1.5 \mathrm{~h}$ of bright light) condition, the phase resetting ability of intermittent bright light exposure, taking into account the reference $-0.31 \mathrm{~h}$ phase shift measured in the control very dim light condition, was 3.2 times greater than that of continuous bright light exposure on a per minute basis of bright light exposure.

The small change in phase observed in the control group may be attributed to the longer-than-24 $\mathrm{h}$ period of the circadian pacemaker (13), and the potentially the weak entraining effect of nonphotic synchronizers (37). We conclude that the additional $2.06 \mathrm{~h}$ average change in phase (DLMOn) observed in the intermittent bright light group as compared to the control group represents the direct effect of the exposure to intermittent bright light on the circadian pacemaker.

Based on previous studies $(3,10,45)$ we expected a robust phase delay for both BL and IBL exposure. Indeed, the dynamic resetting model developed by Kronauer et al. $(30 ; 35 ; 36)$ predicted that the phase shift after IBL would be $\sim 70 \%$ of that after continuous BL. In fact, we found that IBL was $69 \%$ as effective in shifting $\mathrm{CBT}_{\min }, 74 \%$ as effective in shifting $\mathrm{DLMOn}_{25 \%}$, and $83 \%$ and $61 \%$ as effective in shifting $\mathrm{MP}_{25 \%}$ and $\mathrm{DLMOff}_{25 \%}$, respectively compared to BL. For all markers studied, the shifts after IBL exposure were not significantly different from those after BL exposure, and phase delay shifts after IBL and BL exposures were significantly greater than those after VDL exposure. 
The mathematical model developed by Kronauer and colleagues $(32,38,39)$ predicts that there is a greater activation rate (a measure of resetting effectiveness of light) at the very beginning of a light pulse. As activator elements are depleted, the activation rate becomes limited by the rate at which elements are recycled. This model is consequently characterized by two rate parameters: a forward rate, $\alpha$, that depends on light intensity, I, but is fixed at any given intensity, and a backward rate, $\beta$, that is independent of intensity. For any given I, at least two different temporal patterns are needed to estimate $\alpha$ and $\beta$ from the response data $(38,39)$. For $I \cong 10,000 \operatorname{lux}, \alpha$ and $\beta$ were estimated to be $\sim 15 \min ^{-1}$ and $90 \min ^{-1}$ respectively.

For a periodic stimulus pattern consisting of alternating light and dark episodes, we denote that the fraction of cycle time the light is "on" as the "duty cycle". If a window of time is available for stimulation, the maximum response will be generated by using light the entire time (duty cycle=1). However, most of this light is used inefficiently since after a time, $\mathrm{t} \cong \alpha^{-1}$, the incremental response is limited by the recycle rate and consequently is low. The maximally efficient use of a stimulus (i.e., maximum response per minute of stimulus) is found when the duty cycle is very low, but then the total response is also low. Intermediate duty cycles effect a compromise: less than maximum efficiency but total response not a great deal below maximum. The present experiment was designed with several objectives. The first was to expose subjects to a single sequence of bright light pulses, as opposed to multiple sequence of exposure over several days. A second was to extend the range of the duration of near darkness from the former range of 20-45 minutes to 60 minutes. A third was to study a short duty cycle of 0.23 . Both these changes in intermittent light patterns, besides improving the accuracy of rate estimate, increase 
the practical usefulness of intermittent light intervention. Our fourth goal was to validate Kronauer's mathematical model of resetting efficacy of intermittent bright light $(32,38,39)$; simulations based on existing rate estimates indicated that the pattern of light exposure used in this experiment would produce $\sim 70 \%$ of the resetting drive onto the circadian pacemaker achieved by continuous bright light.

These results are in agreement with animal studies that reported that the resetting action of light was most efficient at the beginning of the light exposure, and that minimal additional phase shift was produced by further extension of the light stimulus $(2,42,43,51)$ due to a reduction in photic responsiveness of the circadian pacemaker (35).

It is now recognized that the visual photopic and scotopic systems are not the only photosensitive structures in the retina. Recent studies in mammals have identified a subset of retinal ganglion cells (RGCs) that are intrinsically photosensitive and are involved in conveying photic input to the circadian system $(4,26,28,44,46)$. These cells contain a photopigment melanopsin, that appears to have a broad role in non-visual photoreception (27). Although the involvement of cryptochromes in phototransduction to the circadian system has been suggested (52), recent evidence suggest that these non-visual (melanopsin-containing RGCs) along with the classical visual (rod and cones) photoreceptive systems account for all non-image forming functions involved in pupillary light reflex and circadian photoentrainment $(29,40,44)$. 
Given the unusual light responses of the melanopsin-containing RGCs (4), it is possible that these cells embody the Process L proposed in the model of Kronauer et al. $(38,39)$ that intervenes between light and the pacemaker oscillator (process P). Process L can itself be parsed into two functions. One is a dynamical system in which activation elements are used and subsequently recycled. The other function is a simple power-law relation between the strength of light and the utilization rate parameter. The exponent for the power law inferred for human phase-shift data is proportional to the 0.6 power of light intensity. In their report, Berson et al (4) include data on the acute ( $1 \mathrm{sec}$ average) intracellular depolarization of a ganglion cell as a function of irradiance at several wavelengths of light. A plausible representation of the irradiance dependence is a power law with an exponent of 0.6 over a range of 1.5 to 2 log units (from threshold to saturation). It is possible that these ganglion cells might also embody the dynamical system portion of process $\mathrm{L}$, but the data presented thus far do not cover the temporal range required either to support or to reject this possibility. The response dynamics of rods and cones to light make them unlikely to embody process L. Further investigations, however, including exposures to intermittent light of different duty cycles, and different wavelengths are required to clarify this point.

These findings have important implication as they provide a greater understanding of the effects of brief episodes of light on the human circadian timing system, and suggest that the intermittent exposure to bright light that occurs in everyday life (47) may have a much greater impact on circadian entrainment than previously recognized. The present results also indicate that brief episodes of light exposure may be a cost- and time-effective way of resetting the circadian 
timing system in humans. Such strategies could be used not only treat circadian misalignment related to the low light intensities $(20,54)$ associated with space missions, but also-as shown in the field setting, in which subjects are exposed to conflicting cues-to treat circadian misalignment associated with transmeridian travel, shiftwork $(3,7,10,31)$, and circadian sleep disorders such a advanced (ASPS) and delayed sleep phase syndrome (DSPS). 


\section{ACKNOWLEDGEMENTS}

We thank the subject volunteers, the subject recruiters, the laboratory technicians involved in data collection, and Dr S.W. Lockley for his comments on an early version of the manuscript. This work described in this article was supported by grant NAG 5-3952 from the National Aeronautics and Space Agency, by, and by General Clinical Research Center grant GCRC-M01RR02635 from the National Center for Research Resources. 


\section{REFERENCES}

1. Allan JS and Czeisler CA. Persistence of the circadian thyrotropin rhythm under constant conditions and after light-induced shifts of circadian phase. Journal of Clinical Endocrinology and Metabolism 79: 508-512, 1994.

2. Arvanitogiannis A and Amir S. Resetting the rat circadian clock by ultra-short light flashes. Neuroscience Letters 261: 159-162, 1999.

3. Baehr EK, Fogg LF, and Eastman CI. Intermittent bright light and exercise to entrain human circadian rhythms to night work. American Journal of Physiology 277: R1598-R1604, 1999.

4. Berson DM, Dunn FA, and Takao M. Phototransduction by retinal ganglion cells that set the circadian clock. Science 295: 1070-1073, 2002.

5. Boivin DB and Czeisler CA. Resetting of circadian melatonin and cortisol rhythms in humans by ordinary room light. Neuroreport 9: 779-782, 1998.

6. Boivin DB, Duffy JF, Kronauer RE, and Czeisler CA. Dose-response relationships for resetting of human circadian clock by light. Nature 379: 540-542, 1996.

7. Boivin DB and James FO. Circadian adaptation to night-shift work by judicious light and darkness exposure. Journal of Biological Rhythms 17: 556-567, 2002.

8. Brown EN and Czeisler CA. The statistical analysis of circadian phase and amplitude in constant-routine core-temperature data. Journal of Biological Rhythms 7: 177-202, 1992.

9. Buresova M, Dvorakova M, Zvolsky P, and Illnerova $\mathbf{H}$. Early morning bright light phase advances the human circadian pacemaker within one day. Neuroscience Letters 121: 47-50, 1991.

10. Burgess HJ, Crowley SJ, Gazda CJ, Fogg LF, and Eastman CI. Preflight adjustment to eastward travel: 3 days of advancing sleep with and without morning bright light. J Biol Rhythms 18: 318-328, 2003.

11. Crowley SJ, Lee C, Tseng CY, Fogg LF, and Eastman CI. Combinations of bright light, scheduled dark, sunglasses, and melatonin to facilitate circadian entrainment to night shift work. J Biol Rhythms 18: 513-523, 2003.

12. Czeisler CA, Allan JS, Strogatz SH, Ronda JM, Sanchez R, Rios CD, Freitag WO, Richardson GS, and Kronauer RE. Bright light resets the human circadian pacemaker independent of the timing of the sleep-wake cycle. Science 233: 667-671, 1986.

13. Czeisler CA, Duffy JF, Shanahan TL, Brown EN, Mitchell JF, Rimmer DW, Ronda JM, Silva EJ, Allan JS, Emens JS, Dijk DJ, and Kronauer RE. Stability, precision, and near-24-hour period of the human circadian pacemaker. Science 284: 2177-2181, 1999.

14. Czeisler CA, Johnson MP, Duffy JF, Brown EN, Ronda JM, and Kronauer RE. Exposure to bright light and darkness to treat physiologic maladaptation to night work. New England Journal of Medicine 322: 1253-1259, 1990. 
15. Czeisler CA, Kronauer RE, Allan JS, Duffy JF, Jewett ME, Brown EN, and Ronda JM. Bright light induction of strong (type 0) resetting of the human circadian pacemaker. Science 244: 1328-1333, 1989.

16. Czeisler CA and Waterhouse JM. The effect of light on the human circadian pacemaker. In: Circadian Clocks and Their Adjustment. Chichester (Ciba Found. Symp. 183): John Wiley and Sons, Inc., 1995, p. 254-302.

17. Czeisler CA, Wright Jr KP, Turek FW, and Zee PC. Influence of light on circadian rhythmicity in humans. In: M. New York: Marcel Dekker, Inc., 1999, p. 149-180.

18. Dawson D, Encel N, and Lushington K. Improving adaptation to simulated night shift: Timed exposure to bright light versus daytime melatonin administration. Sleep 18: 11-21, 1995.

19. Dawson D, Lack L, and Morris M. Phase resetting of the human circadian pacemaker with use of a single pulse of bright light. Chronobiology International 10(2): 94-102, 1993.

20. Dijk DJ, Neri DF, Wyatt JK, Ronda JM, Riel E, Ritz-De Cecco A, Hughes RJ, Elliott AR, Prisk GK, West JB, and Czeisler CA. Sleep, performance, circadian rhythms, and light-dark cycles during two space shuttle flights. American Journal of Physiology 281: R1647-R1664, 2001.

21. Drennan M, Kripke DF, and Gillin JC. Bright light can delay human temperature rhythm independent of sleep. American Journal of Physiology 257: R136-R141, 1989.

22. Duffy JF and Dijk DJ. Getting through to circadian oscillators: why use constant routines? Journal of Biological Rhythms 17: 4-13, 2002.

23. Duffy JF, Dijk DJ, Klerman EB, and Czeisler CA. Later endogenous circadian temperature nadir relative to an earlier wake time in older people. American Journal of Physiology 275: R1478-R1487, 1998.

24. Duffy JF, Kronauer RE, and Czeisler CA. Phase-shifting human circadian rhythms: Influence of sleep timing, social contact and light exposure. Journal of Physiology (London) 495: 289-297, 1996.

25. Eastman CI, Liu L, and Fogg LF. Circadian rhythm adaptation to simulated night shift work: Effect of nocturnal bright-light duration. Sleep 18: 399-407, 1995.

26. Gooley JJ, Lu J, Chou TC, Scammell TE, and Saper CB. Melanopsin in cells of origin of the retinohypothalamic tract. Nature Neuroscience 4: 1165, 2001.

27. Gooley JJ, Lu J, Fischer D, and Saper CB. A broad role for melanopsin in nonvisual photoreception. Journal of the Neurological Sciences 23: 7093-7106, 2003.

28. Hattar S, Liao HW, Takao M, Berson DM, and Yau KW. Melanopsin-containing retinal ganglion cells: architecture, projections, and intrinsic photosensitivity. Science 295: 10651070, 2002. 
29. Hattar S, Lucas RJ, Mrosovsky N, Thompson S, Douglas RH, Hankins MW, Lem J, Biel M, Hofmann F, Foster RG, and Yau KW. Melanopsin and rod-cone photoreceptive systems account for all major accessory visual functions in mice. Nature 424: 75-81, 2003.

30. Honma K, Honma S, and Wada T. Phase-dependent shift of free-running human circadian rhythms in response to a single bright light pulse. Experientia 43: 1205-1207, 1987.

31. Horowitz TS, Cade BE, Wolfe JM, and Czeisler CA. Efficacy of bright light and sleep/darkness scheduling in alleviating circadian maladaptation to night work. 281: E384E391, 2001.

32. Jewett ME, Forger Iii DB, and Kronauer RE. Revised limit cycle oscillator model of human circadian pacemaker. Journal of Biological Rhythms 14: 493-499, 1999.

33. Khalsa SBS, Jewett ME, Cajochen C, and Czeisler CA. A phase response curve to single bright light pulses in human subjects. Journal of Physiology (London) 549: 945-952, 2003.

34. Khalsa SBS, Jewett ME, Duffy JF, and Czeisler CA. The timing of the human circadian clock is accurately represented by the core body temperature rhythm following phase shifts to a three-cycle light stimulus near the critical zone. Journal of Biological Rhythms 15: 524$530,2000$.

35. Khammanivong A and Nelson DE. Light pulses suppress responsiveness within the mouse photic entrainment pathway. Journal of Biological Rhythms 15: 393-405, 2000.

36. Klerman EB, Gershengorn HB, Duffy JF, and Kronauer RE. Comparisons of the variability of three markers of the human circadian pacemaker. Journal of Biological Rhythms 17: 181-193, 2002.

37. Klerman EB, Rimmer DW, Dijk DJ, Kronauer RE, Rizzo JF, III, and Czeisler CA. Nonphotic entrainment of the human circadian pacemaker. American Journal of Physiology 274: R991, 1998.

38. Kronauer RE, Forger D, and Jewett ME. Quantifying human circadian pacemaker response to brief, extended, and repeated light stimuli over the photopic range. Journal of Biological Rhythms 14: 500-515, 1999.

39. Kronauer RE, Forger DB, and Jewett ME. Errata Quantifying human circadian pacemaker response to brief, extended, and repeated light stimuli over the photopic range. Journal of Biological Rhythms 15: 184-186, 2000.

40. Lucas RJ, Hattar S, Takao M, Berson DM, Foster RG, and Yau KW. Diminished pupillary light reflex at high irradiance in melanopsin-knockout mice. Science 299: 245-247, 2003.

41. Mills JN, Minors DS, and Waterhouse JM. Adaptation to abrupt time shifts of the oscillator[s] controlling human circadian rhythms. Journal of Physiology (London) 285: 455470, 1978.

42. Nelson DE and Takahashi JS. Integration and saturation within the circadian photic entrainment pathway of hamsters. American Journal of Physiology 277: R1351-R1361, 1999. 
43. Nelson DE and Takahashi JS. Sensitivity and integration in a visual pathway for circadian entrainment in the hamster (Mesocricetus auratus). Journal of Physiology 439: 115-145, 1991.

44. Panda S, Provencio I, Tu DC, Pires SS, Rollag MD, Castrucci AM, Pletcher MT, Sato TK, Wiltshire T, Andahazy M, Kay SA, Van Gelder RN, and Hogenesch JB. Melanopsin is required for non-image-forming photic responses in blind mice. Science 301: 525-527, 2003.

45. Rimmer DW, Boivin DB, Shanahan TL, Kronauer RE, Duffy JF, and Czeisler CA. Dynamic resetting of the human circadian pacemaker by intermittent bright light. 279: R1574-R1579, 2000.

46. Ruby NF, Brennan TJ, Xie X, Cao V, Franken P, Heller HC, and O'Hara BF. Role of melanopsin in circadian responses to light. Science 298: 2211-2213, 2002.

47. Savides TJ, Messin S, Senger C, and Kripke DF. Natural light exposure of young adults. Physiology and Behavior 38: 571-574, 1986.

48. Shanahan TL, Zeitzer JM, and Czeisler CA. Resetting the melatonin rhythm with light in humans. Journal of Biological Rhythms 12: 556-567, 1997.

49. Van Cauter E, Sturis J, Byrne MM, Blackman JD, Leproult R, Ofek G, L'HermiteBal,riaux M, Refetoff S, Turek FW, and Van Reeth O. Demonstration of rapid lightinduced advances and delays of the human circadian clock using hormonal phase markers. American Journal of Physiology 266: E953-E963, 1994.

50. Van Cauter E, Sturis J, Byrne MM, Blackman JD, Scherberg NH, Leproult R, Refetoff $\mathbf{S}$, and Van Reeth $\mathbf{O}$. Preliminary studies on the immediate phase-shifting effects of light and exercise on the human circadian clock. Journal of Biological Rhythms 8: S99-S108, 1993.

51. van den Pol AN, Cao V, and Heller HC. Circadian system of mice integrates brief light stimuli. American Journal of Physiology 275: R654-R657, 1998.

52. Van Gelder RN, Wee R, Lee JA, and Tu DC. Reduced pupillary light responses in mice lacking cryptochromes. Science 299: 222, 2003.

53. Wilmore DW. The metabolic management of the critically ill. New York: Plenum Press, 1977.

54. Wright Jr KP, Hughes RJ, Kronauer RE, Dijk DJ, and Czeisler CA. Intrinsic near-24hour pacemaker period determines limits of circadian entrainment to a weak synchronizer in humans. Proceedings of the National Academy of Science USA 98: 14027-14032, 2001.

55. Youngstedt SD, Kripke DF, and Elliott JA. Circadian phase-delay effects of bright light alone and combined with exercise in humans. 282: R259-R266, 2002.

56. Zeitzer JM, Dijk DJ, Kronauer RE, Brown EN, and Czeisler CA. Sensitivity of the human circadian pacemaker to nocturnal light: Melatonin phase resetting and suppression. Journal of Physiology (London) 526: 695-702, 2000. 


\section{Table 1 legend:}

Timing of Light Exposure Sessions (L.E.S.) and individual phase shifts of the endogenous circadian rhythms of temperature $\left(\mathrm{CBT}_{\min }\right)$ and melatonin $\left(\mathrm{DLMOn}_{25 \%}, \mathrm{MP}_{25 \%}, \mathrm{DLMOff}_{25 \%}\right)$ in the bright light (BL), intermittent light (IBL) and very dim light (VDL) exposure groups. By convention, phase delays are negative (-) shifts, phase advances are positive shifts. Note that due to low amplitude of their CBT rhythms, phase shifts of $\mathrm{CBT}_{\min }$ are not given for subjects 2008, and 2041, and the center of the light exposure sessions relative to $\mathrm{CBT}_{\min }$ is not given for subject 2008. Due to missing blood samples, melatonin $\mathrm{MP}_{25 \%}$ and $\mathrm{DLMOn}_{25 \%}$ could not be calculated for subject 2056. Centers of the light exposure sessions and phase shifts are given in hours. 


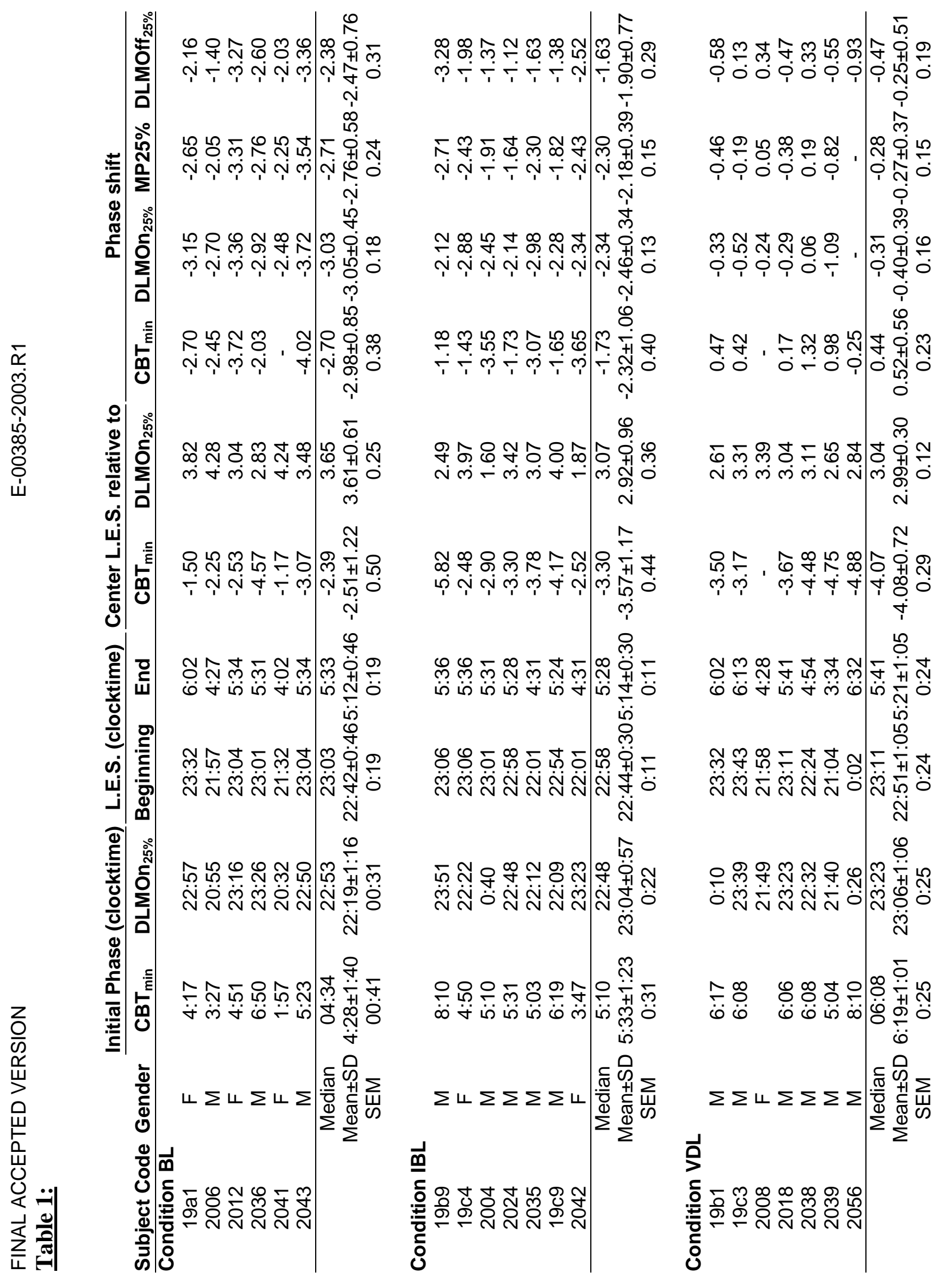




\section{FIGURE LEGENDS}

Figure 1: Raster plot of the 10-day experimental protocol for subject 19a1. Scheduled sleep episodes (8 hours in darkness) are illustrated as black bars. During the baseline days (days 1-3), subjects were exposed to $~ 90$ lux during wakefulness (16 hours). For the remainder of the study, except during the light exposure session, subjects were exposed to $\sim 1.5$ lux during wakefulness (gray bars). A 26.2-h constant routine (CR1) was scheduled on days 4-5. The light exposure session (white bar) was scheduled on days 5-6, and consisted of 6.5 hours of exposure centered 5.8 hours before habitual wake time (i.e., it started $\sim 1.1 \mathrm{~h}$ before habitual bedtime and ended $\sim 2.6 \mathrm{~h}$ before habitual waketime). Following the light exposure day, subjects underwent a 64-h CR (CR2) and were discharged after an $~ 22-h$ recovery sleep episode. Dim light melatonin onsets (DLMOn $25 \%)$ in CR1 and CR2 are indicated by $\Delta$. For this subject, the light exposure (BL condition) generated a phase delay of $3.15 \mathrm{~h}$ in the $\mathrm{DLMOn}_{25 \%}$.

Figure 2: Light exposure conditions: subjects were exposed either to 6.5 hours of continuous bright light (BL; 9,500 lux), intermittent bright light [IBL; six 15-minute bright light ( 9,500 lux) pulses separated by 60 minutes of very dim light (<1 lux)] or very dim light (VDL; <1 lux).

Figure 3: Method used for melatonin phase assessment using Dim Light Melatonin Onset $\left(\right.$ DLMOn $\left._{25 \%}\right)$, Dim Light Melatonin Offset $\left(\right.$ DLMOff $\left._{25 \%}\right)$ and midpoint $\left(\mathrm{MP}_{25 \%}\right)$ phase markers. The thick line represents the three-harmonics fitted curve; the dashed line represents the amplitude of the fitted curve; the dotted line represents $25 \%$ of the amplitude. The figure also illustrates the bright light exposure session (L.E.S.)-induced melatonin suppression. The study 
days corresponding to the profile are given at the top of the figure. CR1: constant routine 1, CR2: constant routine 2 .

Figure 4: Individual resetting responses to the very dim light $(0 \%)$, intermittent bright light $(23 \%)$ and continuous bright light $(100 \%)$ exposure conditions, measured with core body temperature minimum $\left(\mathrm{CBT}_{\mathrm{min}}\right)$ and melatonin phase markers: $\mathrm{DLMOn}_{25 \%}$ (dim light melatonin onset), $\mathrm{MP}_{25 \%}$ (midpoint), and DLMOff $25 \%$ (dim light melatonin offset). Phase-advance shifts are plotted as positive values, whereas phase-delay shifts are plotted as negative values. Horizontal bars represent the median phase shift observed in each condition. 


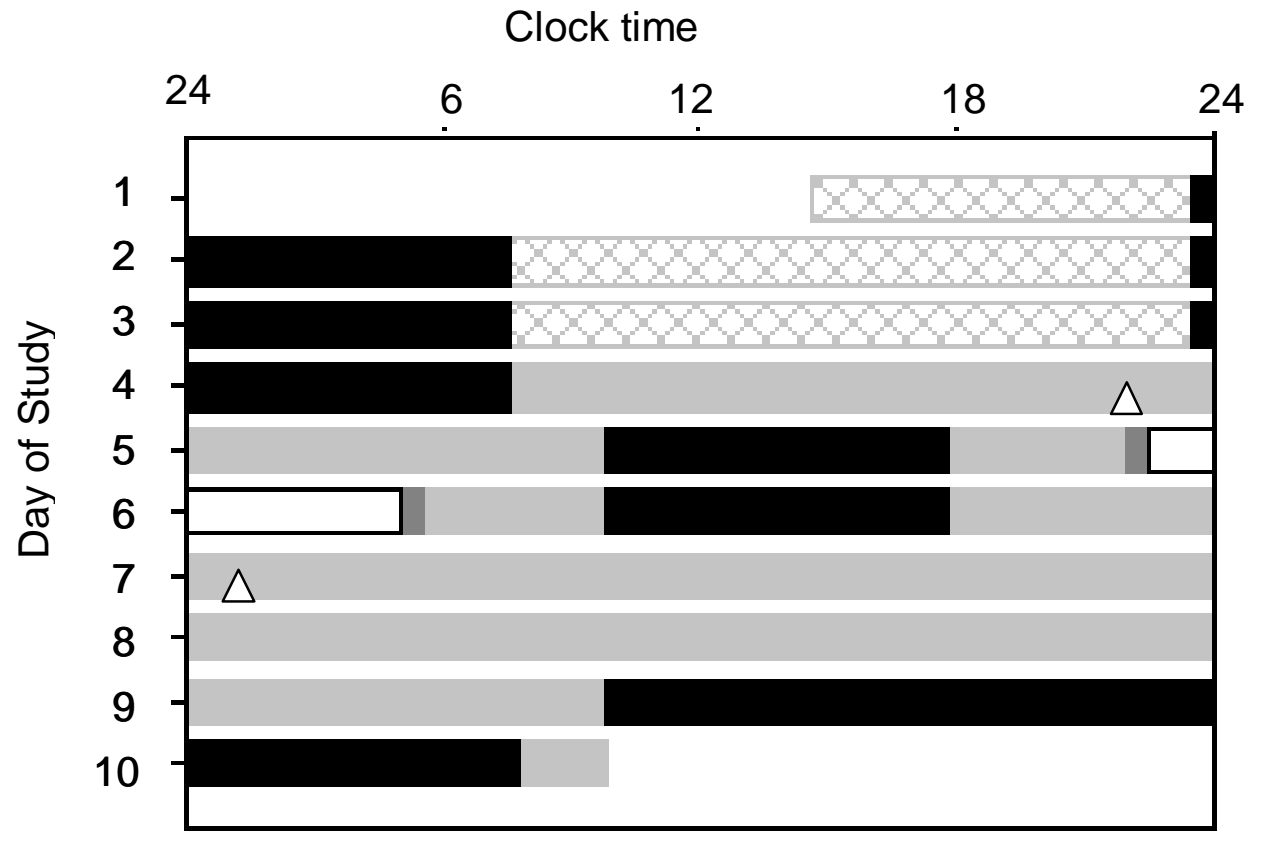

Fig. 1

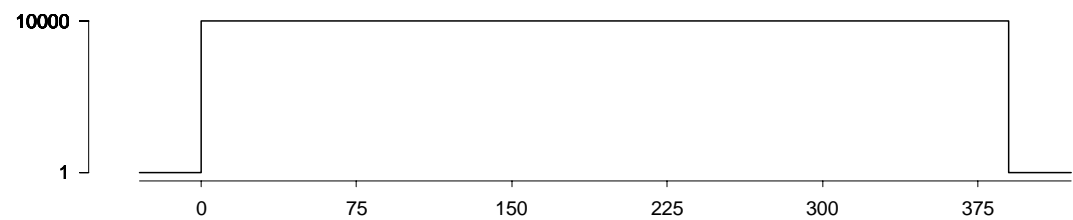

BL
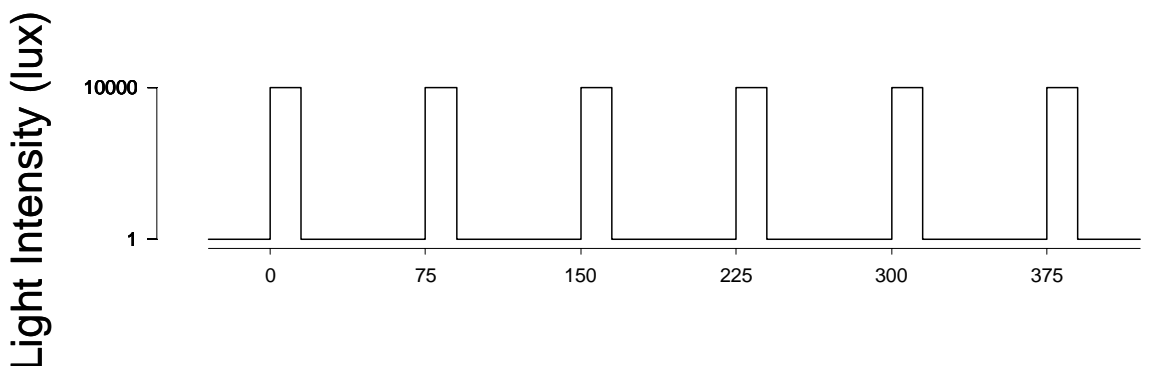

IBL

Fig. 2

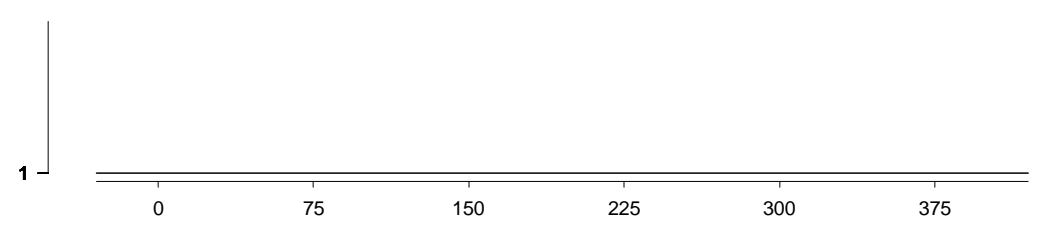

VDL

Time (minutes) 

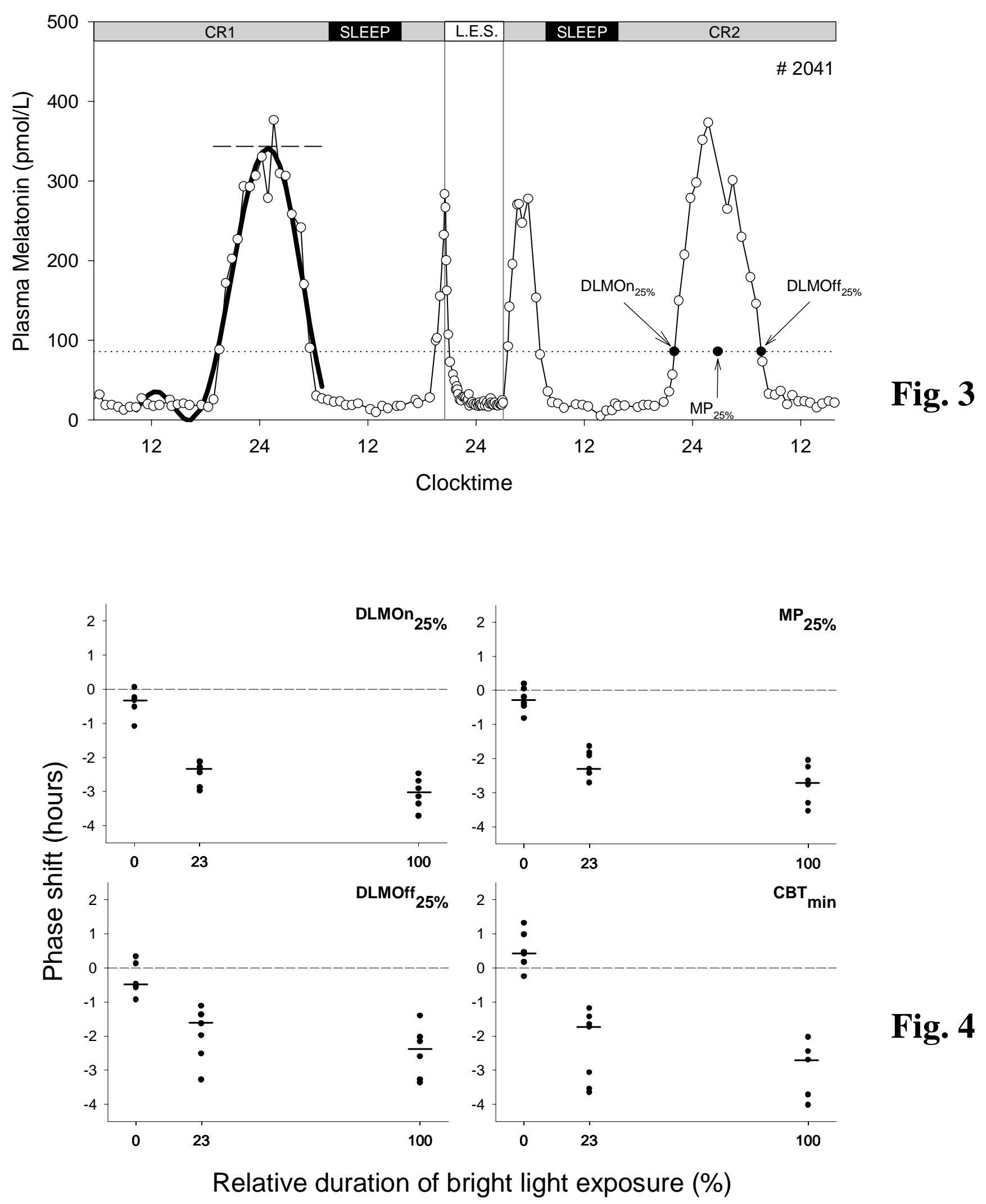